\title{
Avaliação de atividade antioxidante do óleo de Rosa mosqueta (Rosa aff rubiginosa) presente em cosmecêutico para tratamento de feridas
}

Joyce Silva dos Santos ${ }^{1}$, Evalina de Sousa², Maiane de Souza², Lívia Cristina Barreto ${ }^{3}$

${ }^{1}$ Doutoranda em Ciências da Saúde pela Universidade de Brasília, ${ }^{2}$ Mestranda em Ciências da Saúde pela Universidade de Brasília; Professora associada de Ciências Farmacêuticas da Universidade de Brasília.

\section{Introdução}

Rosa aff rubiginosa, popularmente conhecida como Rosa mosqueta é amplamente utilizada na indústria cosmética e alimentícia por sua composição(1).

O óleo fixo extraído por prensagem `a frio de suas sementes é rico em ácidos graxos essenciais à nutrição humana, especialmente ácido linoléico, oléico e linolênico, e ácido trans-retinóico(2,3), além de flavonóides(3), vitamina C e carotenoides(2), substancias eficazes em tratamento cutaneos (4,5). Por esse motivo, tem crescido o interesse por esse insumo para cosméticos com aplicação terapêutica. Contudo, por sua composição majoritariamente graxa, o óleo possui pouca estabilidade devido ao processo natural de peroxidação(6).

Diante do exposto, o presente trabalho visou avaliar a atividade antioxidante do óleo incorporado em formulações cosmecêuticas.

\section{Método}

Foram avaliadas três emulsões, sendo duas contendo $30 \%$ de óleo de Rosa aff rubiginosa, sem ou com BHT (2,6-Di-tert-butyl-p-cresol) como antioxidante sintético, e a terceira formulação elaborada sem a presença do óleo vegetal, porém com BHT, chamada creme branco para fins de comparação. Todas as emulsões, juntamente com o óleo puro foram armazenadas em frascos airless e mantidas em câmara climática por 15 dias à $40 \pm 2^{\circ} \mathrm{C}$ e $30 \pm 5 \%$ de UR (Cf. Fig. 1). 

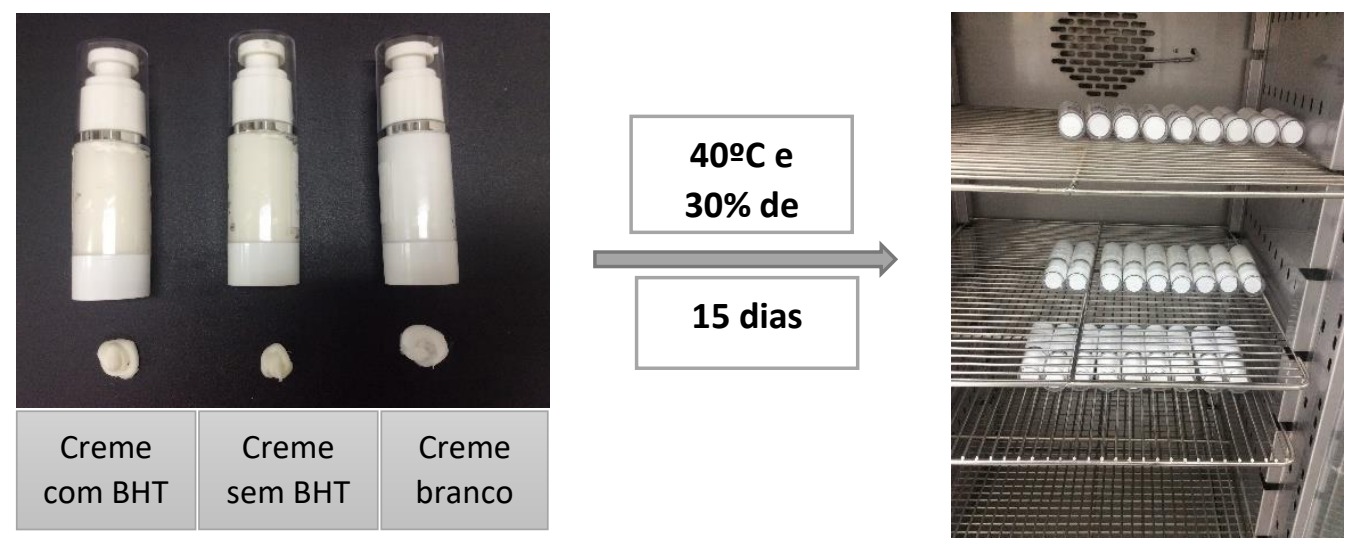

Figura 1 Emulsões desenvolvidas com e sem BHT, juntamente com creme branco (sem óleo de Rosa Mosqueta) acondicionadas em câmara climática.

As amostras recém-preparadas e expostas a tratamento climático por 15 dias foram submetidas a teste de atividade antioxidante pelo método DPPH proposto por Kim et al (2002)(7). A extração de compostos antioxidantes foi efetuada por dissolução de $1,5 \mathrm{~g}$ da amostra em $1,5 \mathrm{~mL}$ de solução de acetona:etanol $(80: 20 \mathrm{v} / \mathrm{v})$. A mistura foi submetida a energia ultrassónica por $15 \mathrm{~min}$, seguido de centrifugação $5000 \mathrm{rpm} / 15 \mathrm{~min}$. Adicionou-se $100 \mu \mathrm{L}$ do sobrenadante obtido a $2900 \mu \mathrm{L}$ do radical $0,1 \mathrm{mM}$ em etanol PA. Após agitação em vórtex, foram mantidos em repouso por $30 \mathrm{~min}$ protegidos da luz. A densidade óptica inicial do radical foi avaliada em espectrofotômetro à absorbância de $517 \mathrm{~nm}$. A atividade antioxidante foi expressa em equivalente Trolox (TEAC).

\section{Resultados e discussão}

A Figura 2 evidencia a ação antioxidante do óleo vegetal, puro ou incorporado em fórmulas cosmecêuticas, como também seu efeito sinérgico em presença de antioxidante sintético. 


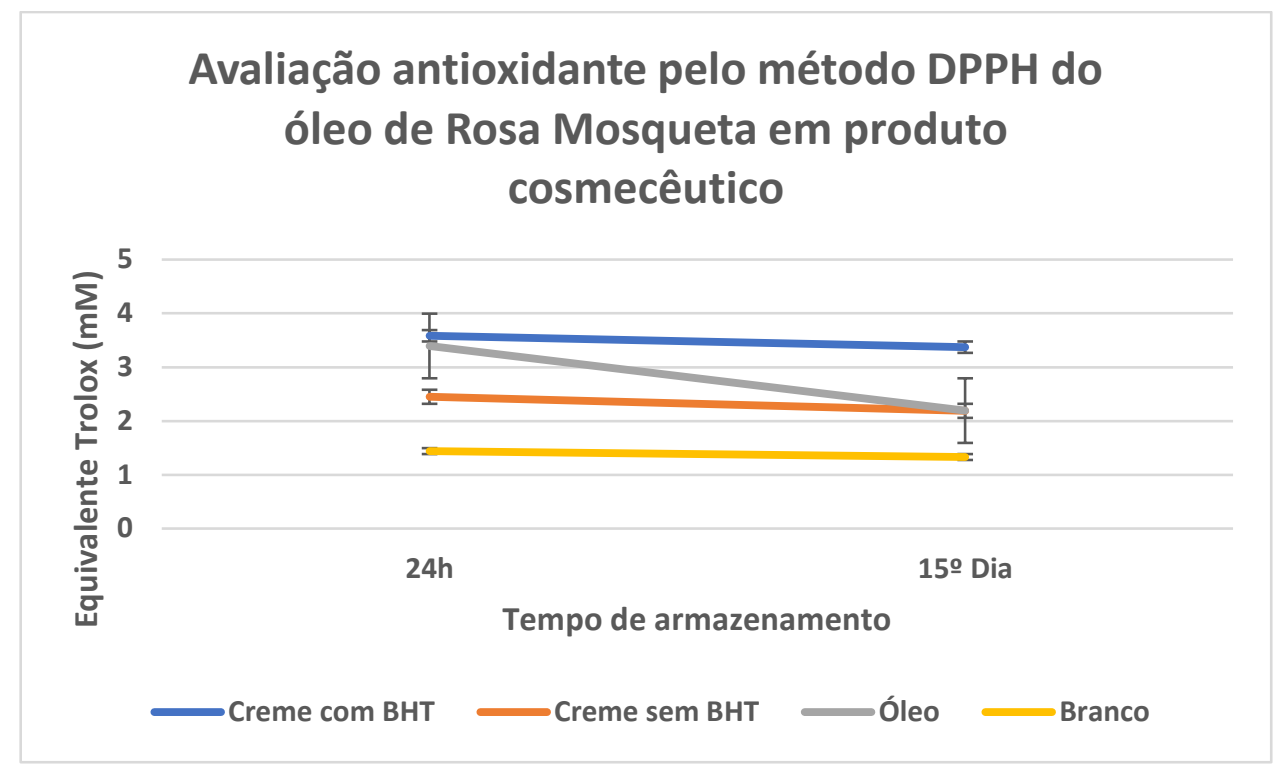

Figura 2 Comparação da atividade antioxidante do óleo incorporado em emulsão contendo ou não $\mathrm{BHT}$ e do óleo puro armazenados a $40^{\circ} \mathrm{C}$ e $30 \pm 5 \%$ de umidade relativa. Fonte: os autores.

Também foi possível observar a manutenção da ação antioxidante, mesmo após tratamento em condições extremas. Entretanto, quando inserido na emulsão, os compostos ativos do óleo foram protegidos do tratamento climático empregado. Outra observação importante diz respeito a ação inferior inicial nas emulsões em comparação ao óleo vegetal, que pode ser justificada pela exposição térmica durante processo produtivo da forma farmacêutica.

Keywords: atividade antioxidante, Rosa mosqueta, óleo vegetal, emulsão, cosmecêutico

\section{Referências:}

1. Valdebenito, G.; Campos, J.; Larrín, O.; Aguilera, M.; Kahler, C.;

Ferrando, M.; García, E.; Sotomayor AG. Innovación tecnológica y comercial de productos forestales no madereros (PFNM) en Chile. 2003.

2. Roman I, Stănilă A, Stănilă S. Bioactive compounds and antioxidant activity of Rosa canina L.biotypes from spontaneous flora of Transylvania. Chem Cent J [Internet]. 2013 Dec 23;7(1):73. Available from: https://bmcchem.biomedcentral.com/articles/10.1186/1752-153X-7-73

3. Fromm M, Bayha S, Kammerer DR, Carle R. Identification and Quantitation of Carotenoids and Tocopherols in Seed Oils Recovered 
from Different Rosaceae Species. J Agric Food Chem [Internet]. 2012 Oct 31;60(43):10733-42. Available from:

https://pubs.acs.org/doi/10.1021/jf3028446

4. Eurides, Duvaldo; Silva, Luiz Antônio Franco; Deleck, Carlos Roberto; Freitas, Patrícia Maria Coletto; Alves LB. Efecto del extracto de óleo de rosa mosqueda (Rosa aff. Rubiginosa) en la cicatrización de heridas cutáneas. Rev electrónica Vet [Internet]. 2011;12(1):1-9. Available from: https://www.researchgate.net/publication/49612023_Efecto_del_extracto_ de_oleo_de_rosa_mosqueda_Rosa_aff_Rubiginosa_en_la_cicatrizacion_ de_heridas_cutaneas_-

_Effect_of_rose_mosqueda_extract_oil_Rosa_aff_Rubiginosa_in_cutane ous_wound_healing

5. Lania BG, Morari J, de Almeida AR, da Silva MN, Vieira-Damiani G, de Almeida Lins K, et al. Topical essential fatty acid oil on wounds: Local and systemic effects. PLoS One. 2019;14(1):1-15.

6. Almeida D.T. de, Viana T.V., Costa M. M., Silva C. de S., Feitosa S. Effects of different storage conditions on the oxidative stability of crude and refined palm oil, olein and stearin (Elaeis guineensis). Food Sci Technol [Internet]. 2019 Jun;39(suppl 1):211-7. Available from: http://www.scielo.br/scielo.php?script=sci_arttext\&pid=S010120612019000500211\&tIng=en

7. Kim J, Noh JH, Lee S, Choi JS, Suh H. Methyl Ether ( TDB ) and Its Antioxidant Activity. Korean Chem Soc. 2002;23(5):661-2. 